\title{
SEQUENTIAL CONTINUITY IN DYADIC SPACES
}

\author{
R. H. MARTY
}

\begin{abstract}
A characterization of metrizability in dyadic spaces in terms of sequential continuity is given. Also, it is shown by examples that invariance under projections (composition) cannot be eliminated from the hypotheses of theorems of Mazur and Engelking concerning the continuity of sequentially continuous functions.
\end{abstract}

The author [3, Theorem 2.9] proved that a dyadic space is metrizable iff it is sequential, and in [6, Problem 4.6] asked whether a dyadic space, for which every sequentially continuous real function defined on a subspace is continuous, must be metrizable. An affirmative answer to this question was announced in [4] and a sharper version is given by Theorem 1.

Let $\left\{X_{\alpha}: \alpha \in A\right\}$ be a collection of topological spaces. For every $y \in X$, the product of the spaces, let $\Sigma(y)=\{x \in X:\{\alpha: x(\alpha) \neq y(\alpha)\}$ is countable $\}$. A dyadic space is a Hausdorff space which is a continuous image of some topological power of the two-point discrete space.

THEOREM 1. Every nonmetrizable dyadic space has a dense, countably compact subspace on which there is defined a sequentially continuous, but noncontinuous, real valued function.

Proof. Let $f: 2^{\kappa} \rightarrow Z$ be continuous and onto and suppose $Z$ is not metrizable. By a result of Efimov [1, p. 248], $f[\Sigma(0)] \neq Z$. Clearly, $\Sigma(0)$ is dense in $2^{\kappa}$ and is countably compact so that $f[\Sigma(0)]$ is dense in $Z$ and is also countably compact. Thus $f[\Sigma(0)]$ is sequentially closed in $Z$. The characteristic function of $f[\Sigma(0)]$ restricted to $f[\Sigma(0)] \cup f[\Sigma(1)]$ (or to $f[\Sigma(0)] \cup\{y\}$ where $y \in Z \backslash f[\Sigma(0)])$ is sequentially continuous but not continuous. This completes the proof.

Let $x^{*}$ be a fixed element of the product $X$ of $\left\{X_{\alpha}: \alpha \in A\right\}$. For every $x \in X$ and every $B \subset A$ we denote by $x_{B}$ the element $(x \mid B) \cup\left(x^{*} \mid A \backslash B\right)$ in $X(x \mid B$ denotes the restriction of $x$ to $B)$. A set $S \subset X$ is said to be invariant under projections provided $x_{B} \in S$ for every $x \in S$ and every $B \subset A$. A set $S \subset X$ is said to be invariant under composition provided $(x \mid B) \cup(y \mid A \backslash B)$ $\in S$ for every $x, y \in S$ and every $B \subset A$. Clearly, $S$ is invariant under composition iff $S$ is invariant under projections for each $x^{*} \in S$. For every $y \in X, \Sigma(y)$ is invariant under composition.

The sets $\Sigma(0)$ and $\Sigma(1)$ are disjoint, sequentially closed subsets of $2^{\kappa}$. Thus

Received by the editors March 14, 1977 and, in revised form, April 12, 1977.

AMS (MOS) subject classifications (1970). Primary 54C05, 54E35; Secondary 54D55.

c American Mathematical Society 1977 
the characteristic function of $\Sigma(0)$ restricted to the subspace $\Sigma(0) \cup \Sigma(1)$ is sequentially continuous but not continuous when $\kappa \geqslant \omega_{1}$. Note that the subset $\Sigma(0) \cup \Sigma(1)$ is not invariant under projections but it is the union of two sets that are invariant under composition.

The subset $\Sigma(0) \cup \Sigma(1)$ relates to theorems of Mazur [5, Theorem III] and Engelking [2, Theorem 4] concerning the continuity of sequentially continuous functions defined on certain subspaces of products.

\section{REFERENCES}

1. B. Efimov, Dyadic bicompacta, Trans. Moscow Math. Soc. 14 (1965), 229-267.

2. R. Engelking, On functions defined on Cartesian products, Fund. Math. 59 (1966), 221-231.

3. R. Marty, m-adic spaces, Acta Math. Acad. Sci. Hungar. 22 (1971) 441-447.

4. __ Metrizability of dyadic spaces, Notices Amer. Math. Soc. 22 (1975), A-653. Abstract \# 75T-G101.

5. S. Mazur, On continuous mappings on Cartesian products, Fund. Math. 39 (1952), 229-238.

6. S. Mrowka, Mazur theorem and m-adic spaces, Bull. Acad. Polon. Sci. Sér. Sci. Math. Astronom. Phys. 18 (1970), 101-107.

Department of Mathematics, Cleveland State University, Cleveland, Ohio 44115. 